\title{
Influence of Solar Minimum on Cosmic Ray Flux, Mutations in viruses and Pandemics like COVID-19
}

\author{
Rajagopal Kamath A \\ Independent Researcher, Kerala, India. Email: rajagopalkamath@gmail.com \\ Charunayan Kamath .R
}

Vellore Institute of Technology, Vellore, Tamilnadu, India email: charunayan@gmail.com

\begin{abstract}
This interdisciplinary study takes into account the effect of the cosmic ray flux on mutations in viruses that result in virulent forms that lead to the occurrence of pandemics. Solar minimum, the reduction in the activity of the Sun which occurs cyclically every 11 years and deep solar minimum that occurs once in a century or so, results in increased cosmic ray flux to the Earth, which in turn generate mutations in viruses harboured in bats and other nocturnal animals. Almost all the previous pandemics occurred during solar minimum years when the Sun spots were lowest or absent and when the solar activity was at the lowest. This study suggests that the present Covid 19 pandemic is triggered by the mutated viromes in bats from latitudes above 30 degrees N. The increase in cosmic ray flux during the solar minimum of solar cycle 24 has contributed to this. It is improbable that SARS-CoV-2 emerged through laboratory manipulation of a related SARS-CoVlike coronavirus. This study indicates that SARS CoV 2 emerged as a result of biological and astrophysical processes.
\end{abstract}

Key words: Cosmic Rays, Solar Minimum, Solar cycle, Sun spots, Geomagnetism, Virome, Mutation, Pandemic, Covid 19, SARS CoV 2.

\section{Introduction}

The cyclical increase in the cosmic rays that arrive onEarth is linked to the changes on the surface of the Sun, mainly the lack of Sun spots. There is a correlation between the cyclical reduction in solar activity termed the solar minimum, which occurs every 11 years and also the grand solar minimum which happen once or twicein a century, and the occurrence of pandemics. During the solar minimum the effect of the solar magnetic field reduces and cosmic rays, the high energy particles from outer space and extra galactic sources, reach the atmosphere. These particles collide with the atoms on the upper part of the atmosphere andresults in secondary particles which shower on the Earth. This activity is more on the night side of the Earth. Bats and other nocturnal animals are reservoirs of many types of viruses. Since they are nocturnal there is more chance of exposure to secondary rays during night time. Moreover, the wings of bats are membranous and entirely different from the wings of birds. The exposure to cosmic ray shower can cause mutation of viruses in bats and other animals. 


\section{Space weather and life forms}

Conditions in space caused by the Sun termed space weather can affect life forms. The magnetic field around the Earth called the magnetosphere extends far out into space.The most visible manifestation of the interaction of the Sun's outer atmosphere and Earth's space magnetospheric environment is the aurora seen in the polar regions. When there is a cosmic ray shower charged particles from space reach the geomagnetic field and affect the upper atmosphere at high latitudes, and above the poles, because that is where the field lines originate. The particles from the Sun that reach the Earth's magnetosphere and ionosphere is variable over a different period.The solar cycle is manifested by the increase and decrease of solar activity as seen in the number of Sunspots. Within the solar cycle, solar storms such as flares and coronal mass ejections are most common within a several-year period known as the solar maximum. Between solar maxima there is a period, called the solar minimum, when the Sun's activity can be extremely low. The present solar cycle 24 began in 2008 with a minimal activity during 2010.The Sun entered the solar minimum phase in 2017. This cycle is expected to end sometime in late 2020. The intensity of cosmic rays at the sea level is not constant. Solar cycle, Sun spot number; solar flares, intensity of the geomagnetic field effect etc affect its value. The intensity is slightly more during night time.Total Solar Irradiance measured from satellites is $\mathbf{1 3 6 5 . 5}$ Watts $/ \mathrm{m} 2$ at solar minimum to 1366.5 Watts $/ \mathrm{m} 2$ at solar maximum. During solar minimum there is a substantial increase in the Cosmic rays, high energy particles whose source is outside the Solar system, reaching the Earth and more in latitudes above 3030 degrees $\mathrm{N}$. The present solar minimum is a deep solar minimum with no Sun spots for a longer period and a greater cosmic ray flux.

\section{Cosmic rays}

Cosmic rays are charged particles and atomic nuclei with energies spanning the range from a few $\mathrm{MeV}$ up to approximately $10^{21} \mathrm{eV}$, which reach the Earth from all directions(1). Cosmic rays affect the living things in many ways and sometimes they can create significant problems. Cosmic rays have the potential to change the genetic material in life forms and affect the chromosomes in reproductive cells.After colliding with the atoms in the upper atmospheric atoms, the cosmic rays generate secondary particles.

\section{Cosmic Rays on Earth since 1964}

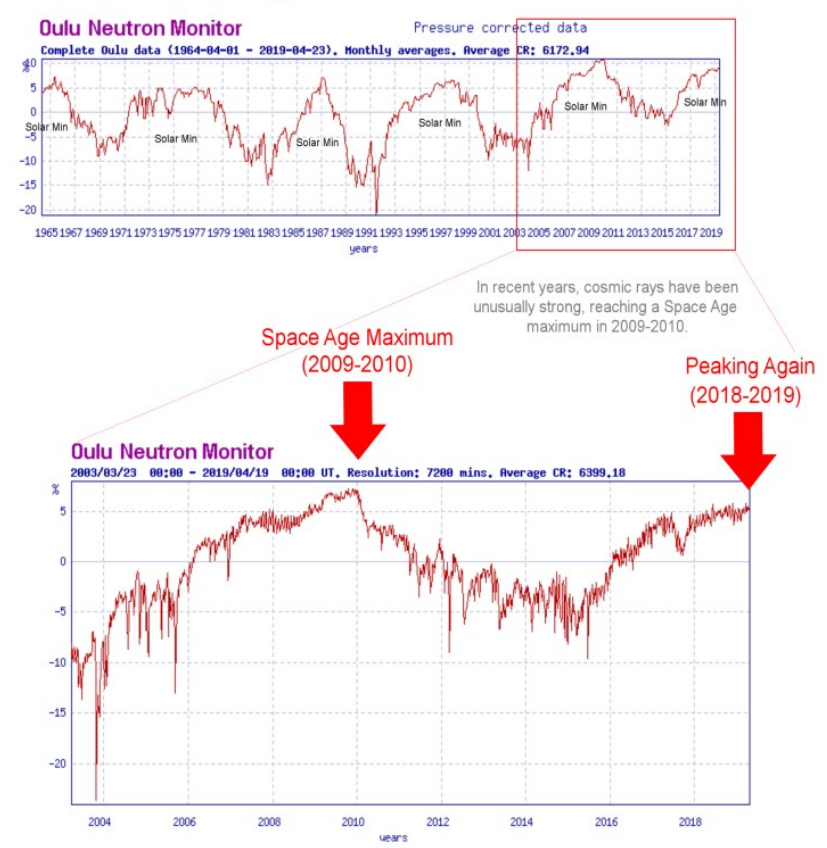

Fig.1: Cosmic ray flux on Earth since 1964.It shows a rising trend now indicating intense cosmic ray shower. Source: spaceweather.com 
Secondary particles spread out and collide with other particles and air molecules which further results in particle shower towards the ground. Secondary particles produced from the primary cosmic rays have iron or other transition elements which makes them move faster. Mutations, the disruption of the genetic material, may be caused by radiation, of which muons are one type. Cosmic rays have the potential to mutate the living cells which come to contact. Genetic mutations are always not harmful but sometimes it can change the animal cells.

Cosmic ray intensity is more during the least number of Sunspots (2). The Sunspot cycles correlate with influenza pandemics associated with the viral mutations. Viruses mutate into a different variety during the solar minima phase when the Sunspots are low.The solar magnetic field is less intense during this phase and allows cosmic ray shower that can change the structure of a virus on impact (2).

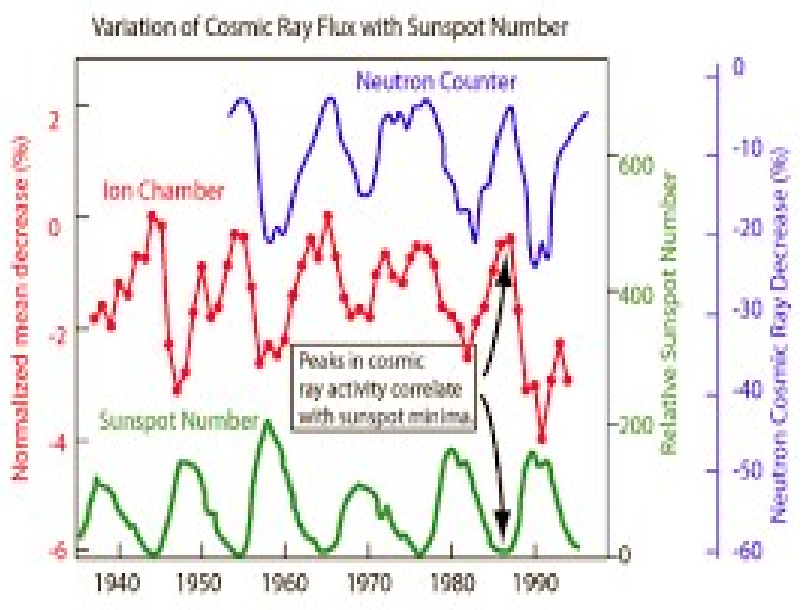

Fig.2: Cosmic Ray Flux and variation in Sunspot number is evident in the picture. Source: NASA

When the Sun is energetic the cosmic rays cause no major problems on Earth. When the Sun is energized, the area around the Sun, the heliosphere, becomes bigger. They get deflected and the arrival of extra galactic cosmic rays is reduced. The cosmic rays have contributed to the mutations that resulted in the evolution of humans and other organisms. It can alter the genetic material in the cells causing new changes in organisms. Every 50 years there will be a supernova explosion in our galaxy. This will cause intense cosmic ray shower.Observations indicate that cosmic rays are coming from all over the galaxy, due to the presence of supernovae(4) in various regions of the galaxy. There are extra galactic sources too. Merger of galaxies, balck holes at the centre of galaxies and other high energy events contribute to the cosmic rays. Occasionally, the intensity of the rays increases. Then a variety of phenomena will occur on the Earth.

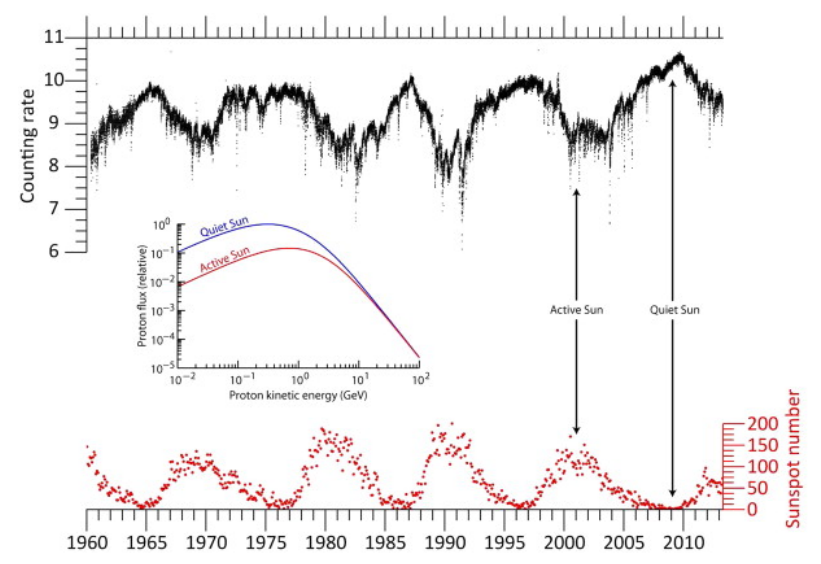

Fig.3: Sunspot number during the past few decades. During solar minimum years Sun spots have reduced in number. Source: NASA

The study of the fossil remains of various oceans, especially the ammonite remains, has suggested the periods of intense radiation that have caused many changes in species and have led to the emergence of new species. When the Sun is hot, the Sun's magnetic field stops the cosmic rays from reaching the earth. During the Maunder Minimum of 1645-1715, the intensity of the cosmic rays was greater. 
During this time, the solar magnetic field was not so strong and the number of Sunspots decreased. These conditions, which caused the Little Ice Age, are due to the fluctuations in the intensity of the cosmic rays. In the early 19th century, the Dalton minimum was characterized by the intensity of cosmic rays.

The cosmic rays constitute particles arrive at the Earth from all parts of the universe. Little is known about the characteristics of this phenomenon and its effects on Earth. Chemical alterations in life forms, mutations, emergence of species, evolution, and climate change are contributed by the effect of cosmic rays. It has been concluded that it also leads to serious health problems in humans (3). These are harmful to living things as they are the particles that cause ionization. Those living in the highlands and on frequent flights often take an extra dose of these rays. Cosmic radiation can cause genetic and cytogenetic damage. Cosmic rays cause an increase in cancer rates (3). Mutations cause a variety of defects in the next generation since changes in embryonic cells may occur.The scientific world has now received astounding evidence of the changes they are making on Earth. These particles arrive on the Earth after travelling millions of light years. In the cosmic rays, there are many atomic nuclei. The most common is helium atom. It has two protons and two neutrons. The rays have the nuclei of carbon, oxygen and iron, as well as a few other elements. Protons and electrons are also showered. There are antimatter particles, positrons, and particles that we have not yet identified. We do not yet know the exact source of these particles. It is harmful to living organisms due to its high energy. But the Earth's atmosphere acts as a shield and prevents these particles from reaching the surface directly. The geomagnetic field also provides protection.

Particles in cosmic rays that collide with the Earth's atmosphere collide with atoms. This results in many secondary particles. These subunits again collide with the atoms in the atmosphere to form new particles. Multiple collisions cause the energy of the cosmic rays to be distributed. The arrival of these particles is known as 'air shower'(5). When an ion collides with atmospheric particles, at least fifty secondary particles are formed. They then collide again and give birth to more particles. The ones with the highest energy are few. There are muons, neutrinos and also a few neutrons. Some particles, which have the energy of a small stone that is thrown, reach the Earth's surface. There are objects in the universe capable of delivering several times more energy to a proton than the energy we have created in the particle laboratories so far. Most of these rays collide with atoms in the atmosphere and disintegrate. This means that particles with very high energies disintegrate in the Earth's atmosphere. Due to the continuous collisions with atmospheric particles secondary and tertiary low-energy rays reach the Earth's surface. 

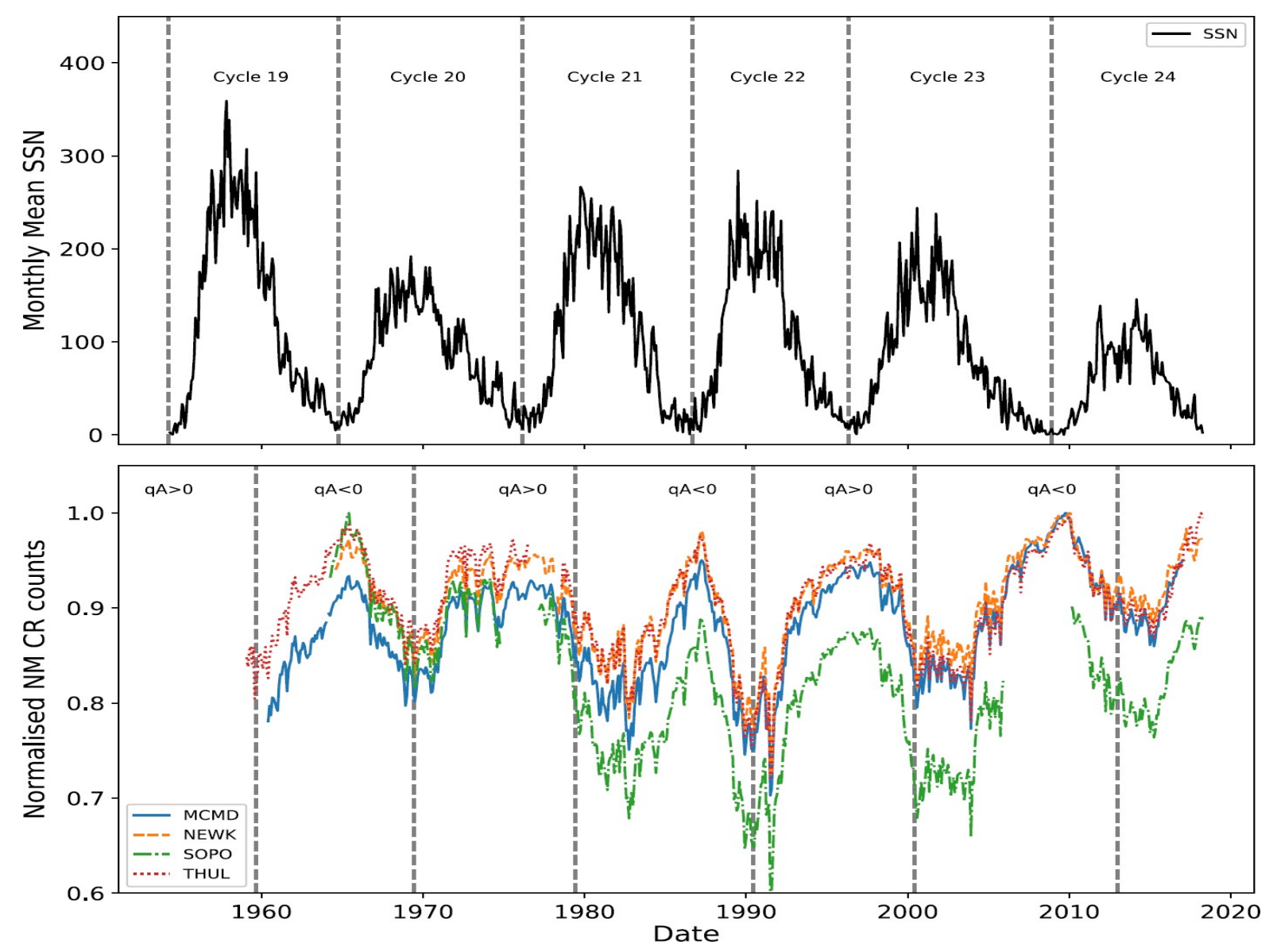

Fig .4: Sun Spot number SSN with vertical lines showing the beginning of each solar cycle. Cosmic ray CR intensity, with vertical lines showing the approximate epochs of solar magnetic-field polarity (Image courtesy: Ross, E., Chaplin, and W.J. The Behavior of Galactic Cosmic-Ray Intensity during Solar Activity Cycle 24. Sol Phys 294, 8 (2019).

\section{Sun and the pandemics}

During the period 2007-2020 the Sun is at deep solar minimum stage which has resulted in increased cosmic ray flux. These rays have generated mutation in bats in the surroundings of Wuhan, China. The below given pandemics occurred during solar minimum years when the Sun spot numbers were very low or absent and the cosmic ray flux was maximum. The Black Death plague: 1346-1353,Great Plague of London: 1665, Great Plague of Marseille, France: 1720-1723, Russian Plague: 1770-
1772,Flu pandemic: 1889-1890, ,Spanish Flu: 1918-1920,Asian Flu: 1957-1958,H1N1 Swine Flu pandemic: 2009-2010 and COVID 19 pandemic 2019-2020 6),(7) happened during solar minimum periods.Last 400 years' data was analyzed for recording the changes in the number of sunspots. It was found that the numbers of sunspots were too low or almost absent during solar minimum years. Starting 1645 to 1715, during the Maunder Minimum period, there was little solar activity and very few sunspots. During 1917-1920, 2009-2010 and starting 2017 the Sun has been in the solar 
minimum stage with least number of Sun spots.

\section{Corona Virus}

Coronaviruses (CoVs) are of the subfamily Orthocoronavirinae of the family Coronaviridae, order Nidovirales. CoVs have an envelope, crown-like part. The $\mathrm{CoV}$ genome is single-strand RNA $27-32 \mathrm{~kb}$ in size, the second largest of all RNA virus genomes. The size of CoVs is associated with increased replication, acquiring genes by encoding RNA-processing enzymes. Genome expansion facilitates the CoVs to adapt to hosts. CoVs cause disease in domestic and wild animals and in humans (8]. The $\alpha$ - and $\beta$ CoVs mainly infect mammals and $\gamma$ - and $\delta$ CoVs mainly infect birds (8) By the process of recombination, gene interchange, and gene insertion or deletion genome changes occur in CoVs. Highly pathogenic SARS-CoV and MERS-CoV have caused pandemics in humans. Present pandemic of Covid 19 is caused by SARS-CoV 2 virus.SARS-CoV-2 is the seventh coronavirus known to infect humans; SARS-CoV, MERSCoV and SARSCoV-2 can cause severe disease, whereas HKU1, NL63, OC43 and 229E are associated with mild symptoms (9)

\section{Bats}

The SARS-CoVshas been reported in some species of horseshoe bats in the genus Rhinolophus. (9) Horseshoe bats are small, with forearm lengths of $30-75 \mathrm{~mm}$ and combined head and body lengths of 35$110 \mathrm{~mm}$. Majority of the species have long, smooth fur. They get their common name from their large nose-leafs, which are shaped like horseshoes. The natural reservoirs for a number of emerging zoonotic viruses are Bats. These flying mammals carry henipaviruses, corona viruses and variants of rabies viruses. Some horseshoe bat species (genus Rhinolophus) have a large number of viruses that have a genetic similarity with the coronavirus associated with severe acute respiratory syndrome (SARS)(10).

The discovery of bat SARS-like coronaviruses and the diversity of coronaviruses in bats have given new insights on the origin and transmission of SARS like corona viruses. Genome sequencing has indicated that the SARS-CoV is a new virus which is not having any genetic relation to any other identified human coronaviruses The emergence of zoonotic viruses from wildlife requires interspecies contact, cross-species virus transmission, and the adaptation of the within the spillover species. Three zoonotic coronaviruses have been identified as the cause of large-scale disease outbreaks-Severe Acute Respiratory Syndrome (SARS), Middle East Respiratory Syndrome (MERS), and Swine Acute Diarrhea Syndrome (SADS). SARS and MERS emerged in 2003 and 2012, respectively, and caused a worldwide pandemic.

\section{Mutations}

The SARS-CoV-2

genome has a RNA molecule of about 30,000 bases containing 15 genes, including the $\mathrm{S}$ gene which codes for a protein on the surface of the viral envelope. SARS-CoV-2 belongs to the group of Betacoronaviruses, very close to SARS-CoV.The viruses isolated from a bat of the species Rhinolophus affinis has recently been described as very similar to SARS-CoV2 , with genome sequences identical to $96 \%$. 
These results indicate that bats, and in particular species of the genus Rhinolophus, are the reservoir of the SARS-CoV and SARS$\mathrm{CoV}-2$ viruses. The coronavirus isolated from pangolins is similar to the above virus. SARSCov-2 virus is the result of a recombination of genetic material of two different viruses (11).

Echolocating bats generate ultrasound and emit the sound through the open mouth or, much more rarely, the nose. The latter is most pronounced in the horseshoe bats. Bat echolocation range in frequencies from 14,000 $\mathrm{Hz}$ to over $100,000 \mathrm{~Hz}$. The secondary cosmic ray shower arising out of the increased cosmic ray flux during the solar minimum interferes with the echolocation exercise. Moreover, the cosmic ray shower is more during night time and since the bats are nocturnal, millions of flying bats act as receivers for cosmic rays in the latitudes above $30 \mathrm{~N}$. Most of the virus carriers are nocturnal animals like bats, shrews, civets, and pangolins. Cosmic ray particles can penetrate the bat wings which are membranous, unique to these life forms. Sudden impact of cosmic secondary rays can initiate mutations in microbes in these animals. The preexisting SARS CoV virus in one of the bats would have mutated to a different one by way of impact from a high energy particle from the cosmic ray flux. Many such mutations would have occurred. The most virulent form has jumped from bats to an intermediate host.

The fecal-oral route may be the main mode of transmission among animals. The mixing of reservoir hosts like bats and intermediate hosts like pangolins/civets would be responsible means of transmission. The cross-species transmission in the market may have occurred from contaminated feces, urine, blood, or aerosols. This may also be true for pangolin/civet-to-human transmission. We should construe that these types of events would have occurred during the SARS Co V 2 outbreaks and contributed to the spread of Covid 19 around the world.

\section{Conclusion}

The solar cycle that started in 2008 is ending in 2020 with a solar minimum. This is a deep solar minimum with least number of Sun spots. Such deep minimums occur once or twice in a century. The cosmic ray flux on Earth has increased and so is the secondary shower from the colliding cosmic rays above the atmosphere. The high energy heavy particles cause mutations in the life forms on the Earth. The recombination of two earlier viruses in bats has occurred during the solar minimum phase starting 2017. The interference of cosmic rays with the echolocation excersice in bats has exacerbated the process of mutation of viruses harboured in them. As in almost all earlier pandemics, the increase in cosmic ray flux from the reduced solar activity has initiated the mutation in viruses in bats and has resulted in new viral strands.If SARS-CoV-2 pre-adapted in another animal species, then there is the risk of future re-emergence events. In contrast, if the adaptive process occurred in humans, then even if repeated zoonotic transfers occur, they are unlikely to take off without the same series of mutations.In addition, identifying the closest viral relatives of SARS-CoV-2 circulating in animals will greatly assist studies of viral function. The evidence shows that SARS CoV-2 is not a manipulated virus and has emerged from natural causes. 


\section{References}

1) Giacalone, J. Energetic particle transport. In Heliophysics: Space Storms and Radiation: Causes and EffectsCambridge University Press(pp. 233-262).

2) Rossella Di Trolio, Giuseppe Di Lorenzo, BrunoFumo, Paolo A AsciertoFUTURECosmic radiation and cancer: is there a link? Future Oncology, VOL11, NO. 725 Mar 2015

3) Amato, E, The origin of galactic cosmic rays. International Journal of Modern Physics D, Vol23, No. 07, 1430013 (2014).

4) Radio detection of Cosmic-Ray Air Showers an,d High-Energy Neutrinos Frank G. Schröder arXiv:1607.08781

5) Wickramasinghe NC, Edward J Steele, and Wainwright M, GensukeTokoro Manju Fernando and Jiangwen Qu, Sunspot Cycle Minima and Pandemics: The Case for Vigilance? Journal of Astrobiology \& Outreach, 5:2,2017

6) K F TappingRichard G MathiasD L Surkan, Influenza pandemics and solaractivity, Canadian Journal of Infectious diseases, Jan 2001.

7) Susanna K. P. Lau and Chan Coronaviruses: emerging and re- emerging pathogens in humans and animals Virology Journal, 12:209,2015

8) Lau SK, Woo PC, Li KS, Huang Y, Tsoi HW, Wong BH, et al. Severe acute respiratory syndrome coronavirus-like virus in Chinese horseshoe bats. PNAS. 102:14040-5, 2005.

9) $\mathrm{Hu}, \mathrm{B} ., \mathrm{Ge}, \mathrm{X}$., Wang, L. et al. Bat origin of human coronaviruses. Virol J 12, 221 (2015).

10) Gaisser, Tom, Cosmic Rays and Particle Physics. (Cambridge Univ. Press, 1991)

11) Zheng J. SARS-CoV-2: an Emerging Coronavirus that Causes a Global Threat. Int J Biol Sci. 2020; 16(10):1678-1685.

12) Cronin, Gaisser and Swordly, "Cosmic Rays at the Energy Frontier" Scientific American, January 1997,pp. 44-49

13) Rossi, Bruno, High Energy Particles. Prentice-Hall, 1952.

14) Friedlander, Michael W. Cosmic Rays, Cambridge, MA, Harvard University Press, 1989, 166 p. 